This is the submitted version of the article:

Sánchez-Ochoa F., Hidalgo F., Pruneda M., Noguez C.. Unfolding method for periodic twisted systems with commensurate Moiré patterns. Journal of Physics Condensed Matter, (2020). 32.

025501: - . 10.1088/1361-648X/ab44f0.

Available at: https://dx.doi.org/10.1088/1361-648X/ab44f0 


\title{
Unfolding method for periodic twisted systems with commensurate Moiré patterns
}

\author{
F. Sánchez-Ochoa ${ }^{1}$, Francisco Hidalgo $^{2,3}$, Miguel Pruneda ${ }^{3}$ \\ and Cecilia Noguez ${ }^{1}$ \\ 1 Instituto de Física, Universidad Nacional Autónoma de México, Apartado \\ Postal 20-364, Cd. de México C.P. 01000, Mexico \\ 2 Departamento de Ciencias Básicas, Univerisdad Autónoma \\ Metropolitana-Azcapotzalco, Av. San Pablo 180, Cd. México C.P. 02200, \\ Mexico \\ 3 Catalan Institute of Nanoscience and Nanotechnology (ICN2), CSIC and \\ BIST, Campus UAB, Bellaterra, 08193 Barcelona, Spain \\ E-mail: fsanchez@fisica.unam.mx, cecilia@fisica.unam.mx
}

\begin{abstract}
.
We present a general unfolding method for the electronic bands of systems with double-periodicity. Within density functional theory with atomic orbitals as basis-set, our method takes into account two symmetry operations of the primitive cell: a standard expansion and a single rotation, letting to elucidate the physical effects associated to the mutual interactions between systems with more than one periodicity. As a result, our unfolding method allows studying the electronic properties of vertically stacked homo- or heterostructures. We apply our method to study $(3 \times 3)$ single-layer graphene, $(\sqrt{13} \times \sqrt{13})$ twisted single-layer graphene, and $(2 \sqrt{3} \times 2 \sqrt{3})$ graphene- $(\sqrt{7} \times \sqrt{7})$ tungsten disulfide heterostructure with an interlayer angle of $10.9^{\circ}$. Our unfolding method allows observing typical mini gaps reported in heterostructures, as well as other electronic deviations from pristine structures, impossible to distinguish without an unfolding method. We anticipate that this unfolding method can be useful to compare with experiments to elucidate the electronic properties of two-dimensional homo- or heterostructures, where the interlayer angle can be considered as an additional parameter.
\end{abstract}

Keywords: graphene, tungsten disulfide, heterostructures, twisted bilayers, DFT, unfolding 


\section{Introduction}

Vertically stacked two-dimensional (2D) systems with van der Waals (vdW) interactions have fascinating physical properties and potential applications in optoelectronics, electronic transport [1], among other fields. In principle, these $2 \mathrm{D}-\mathrm{vdW}$ structures should preserve the physical advantages of their pristine monolayers but also allow for achieving unusual and even superior properties, which cannot be obtained otherwise [2]. Besides the atomic composition of each monolayer, the vertical stacking adds a new parameter: the interlayer rotation angle $(\theta)$. In general, when two vertically stacked monolayers of the same composition (a homostructure) have a $\theta \neq 0$, a Moiré pattern is formed, being its commensurable area inversely proportional to $\theta$ [3]. In contrast, two vertically stacked monolayers of different atomic composition (heterostructure) also exhibit a Moiré pattern just by the condition of a lattice mismatch of layers [4]. In this sense, different theoretical and experimental groups have reported new physical properties exhibited by two vertically stacked graphene $(\mathrm{G})$ layers related to $\theta$, including optical activity[5], topological edge states[6], and a Mott insulator-to-superconductor transition at a critical $\theta[7,8]$. Besides bilayer $\mathrm{G}$, the vertical stacking of transition metal dichalcogenides (TMDCs) with $\mathrm{G}$ have been considered promising materials for the design of new electronic, optoelectronic, and photonic devices, in particular based on tungsten disulfide $\left(\mathrm{WS}_{2}\right)$ or molybdenum disulfide $\left(\mathrm{MoS}_{2}\right)[5,9,10,11$, $12,13]$, both layered semiconductors with a direct band gap for single layer.

Through first-principles methods, for instance, based on Density Functional Theory (DFT), it is possible to study the electronic properties of these novel 2D materials, where the use of periodic boundary conditions is highly desirable to reduce the computational effort. However, periodic boundary conditions require matching of the lattice constants of vertically stacked layers to construct a commensurate supercell (SC) of the bilayer heterostructure, such that each layer can be built by a conventional or non-conventional SC. By definition, a conventional $\mathrm{SC}$ is constructed just by expansion symmetry operations on the primitive cell (PC), while nonconventional SC requires expansion and rotation operations. In consequence, SC method is a common practice in periodic DFT calculations, making available the study of systems with thousands of atoms, different composition, impurities, vacancies, or combined systems, including vertically stacked 2D$\mathrm{vdW}$ heterostructures with $\theta \neq 0$.

Nevertheless, the use of SCs leads to an artificial folding of the electronic bands as a consequence of the reduction of the reciprocal space [14]. Such folding effects hide the real physical interactions, usually leading to a misinterpretation of the results in the Brillouin zone (BZ) of the SC. Indeed, the comparison between the theoretical electronic band structure of SC and angle-resolved photoemission spectroscopy (ARPES) measurements is quite difficult [15]. A successful technique to unveil the change of electronic structure, hidden by the use of SC method, is the band unfolding approach (hereafter, unfolding for simplicity), which have been developed as a postprocessing tool for DFT [16, 17, 18, 19, 20, 21] and tight-binding calculations as well [22, 23, 24]. In the unfolding method for DFT, the calculated Kohn-Sham orbitals of $\mathrm{SC}$ are unfolded into the original $\mathrm{BZ}$ of the $\mathrm{PC}$. This technique projects the electronic states from the $\mathrm{BZ}$ of the $\mathrm{SC}$ to the $\mathrm{BZ}$ of the reference system by assigning a spectral weight, $W_{k}(I)$, where $k$ is a point on the reciprocal space of the SC and $I$ denotes $I$-th electronic band to be unfolded. Consequently, $W_{k}(I)$ quantifies how much of the reference system remains in the perturbed one, for example, when the translation symmetry is lost. However, unfolding methods for DFT calculations have been applied only for conventional hexagonal SCs [20, 21, 25]. In contrast, unfolding methods for tightbinding calculations have been improved for hexagonal and non-conventional SCs, and applied to twisted systems [16, 26].

Therefore, we present an unfolding method to analyze the electronic structure of $2 \mathrm{D}$-vdW twisted systems under the scheme of DFT with atomic orbitals as basis-set. Our method is an extension of previous work of [27] and [28], allowing the unfolding of electronic bands of structures constructed by conventional and non-conventional SCs. To show the potentiality of the method, we investigate the electronic band structure of two ideal G single-layer with periodicities $3 \times 3$ and $\sqrt{13} \times \sqrt{13}$. Furthermore, the electronic band structure of a twisted G-WS bilayer with $\theta=10.9^{\circ}$ is unfolded using our method, unveiling the physical consequences of the mutual interactions between $\mathrm{G}$ and $\mathrm{WS}_{2}$ layers. The content of the paper is organized as follows. In section 2, we present a brief description of the unfolding method. Section 3 shows the models studied here to validate our method. Section 4 summaries the computational details employed in the DFT calculations. Then, in Section 5, we use our method to unfold the electronic band structure of the SCs described above. Finally, in section 6 we discuss our main conclusions.

\section{Method}

Under the scheme of DFT with a linear combination of atomic orbitals (LCAO) as basis-set and using Bloch's 
theorem, the total wavefunction of a system is:

$\psi_{I}(\mathbf{k}, \mathbf{r})=\sum_{j^{\prime}}\left(\operatorname{expik} \cdot \mathbf{R}_{\mathbf{j}^{\prime}}\right) \phi_{j^{\prime}}(\mathbf{r}) c_{j^{\prime} i}(\mathbf{k}) ;$

where the sum in $j^{\prime}$ extends to all basis orbitals in space, $I$ labels the different bands, and $c_{j^{\prime} i}$ corresponds to the expansion coefficients of the Kohn-Sham orbitals obtained by the self-consistent calculation. The base of our unfolding method stands on the calculation of the spectral weight, $W_{k}(I)$ which quantifies the projection of the electronic state from the $\mathrm{BZ}$ of the $\mathrm{SC}$ to the $\mathrm{BZ}$ of the PC for the I-th band. Hence, following the development of Deretzis [27], $W_{k}(I)$ is obtained as:

$W_{k}(I)=\frac{1}{\mathcal{N}} \sum_{u \in P C}\left[\sum_{j}^{\mathcal{N}} \tilde{c}^{u+j}(k)\right] \times\left[\sum_{j}^{\mathcal{N}} c^{u+j}(k)\right]$,

where the sum on $c$ coefficients run over all the equivalents orbitals $j$ of the $\mathcal{N}$ times repeated PC, and over all atoms $u$ with equivalent positions in SC with respect to PC. Here, $c$ is a complex number and $\tilde{c}$ represents its complex conjugate, and $\mathcal{N}$ is an integer defined as $\mathcal{N}=\Omega_{S C} / \Omega_{P C}$, where $\Omega$ denotes the volume in the real space.

Notice that $W_{k}(I)$ determines the equivalence of the electronic states of $\mathrm{SC}$ on the corresponding of $\mathrm{PC}$ and must obey that $0 \leq W_{k}(I) \leq 1$, which is true if and only if the basis-set is orthogonal and normalized [27, 28]. For SC with more than one periodicity or built up by more than one $\mathrm{PC}$, for instance, any vertically stacked heterostructure, it is necessary to define on which $\mathrm{PC}$ the electronic states will be projected. A wrong choice on the PC could induce a bad interpretation on the unfolded bands or even ghost states [26]. Once the PC has been defined, the problem of identify the equivalent $u$ atoms in SC is directly solved because the layer was constructed by expansion and rotation symmetry operations on the PC, which involves an affine transformation between SC and PC. This transformation consists of two parts: a shift of the origin and a linear part. The former is given just by a straight translation of the lattice vectors of PC $(\mathbf{a}, \mathbf{b}, \mathbf{c})$, while the latter implies a change of their orientation, length or both. Through just by a linear transformation, the lattice vectors of the $\mathrm{SC}\left(\mathbf{a}^{\prime}\right.$, $\left.\mathbf{b}^{\prime}, \mathbf{c}^{\prime}\right)$ are defined by:

$\left(\mathbf{a}^{\prime}, \mathbf{b}^{\prime}, \mathbf{c}^{\prime}\right)=(\mathbf{a}, \mathbf{b}, \mathbf{c}) \mathbf{P}$,

with $\mathbf{P}$, a transformation matrix, defined as:

$\mathbf{P}=\left(\begin{array}{lll}P_{11} & P_{12} & P_{13} \\ P_{21} & P_{22} & P_{23} \\ P_{31} & P_{32} & P_{33}\end{array}\right)$,

where $P_{m n}$ are positive integer values and the absolute value of the determinant of $\mathbf{P}$ corresponds to $\mathcal{N}$. Notice that $P_{m n}$ can be parametrized in terms of two or three integer values whether the transformation is carried out in $2 \mathrm{D}$ or $3 \mathrm{D}$, respectively. Therefore, the equivalent $u$ atoms of the $\mathrm{SC}$ from $\mathrm{PC}$ is easily obtained through Eq. (3) and Eq. (4).

For 2D hexagonal lattice, any conventional or nonconventional SC can be set up using the transformation matrix $\mathbf{P}$, considering $\mathbf{c}$ vector as an invariant [29]. Thus, $P_{m n}$ elements in Eq. (4) are $P_{33}=1$ and $P_{13}=$ $P_{23}=P_{31}=P_{32}=0$. Finally, the other elements are functions of two integer parameters, $m$ and $n$ to take into account expansion and rotation operations. Then, the matrix transformation, $\mathbf{P}$, simplifies to:

$\mathbf{P}=\left(\begin{array}{ccc}m & n & 0 \\ -n & m+n & 0 \\ 0 & 0 & 1\end{array}\right)$

Notice that if $m$ or $n=0$, then the 2D hexagonal SC is a conventional one, while a non-conventional $\mathrm{SC}$ is obtained for $m, n \neq 0$. For our propose, given a $2 \mathrm{D} \mathrm{SC}$, it is necessary to identify which of the atomic positions are equivalent to the PC. This is solved by a process which follows the inverse process described above to set up a SC from a PC. Additionally, because commonly DFT calculations are carried out including a structural relaxation, it is necessary to specify a threshold value to determine whether an atom has deviated from its initial position or not.

Another important issue for the calculation of $W_{k}(I)$ is the scanning of the BZ along specific paths of the $\mathrm{SC}$ and their projections on equivalent paths of the $\mathrm{BZ}$ of $\mathrm{PC}[27,28]$. In general, the fractional coordinates $\left(k_{x}, k_{y}, k_{z}\right)$ of any point in the reciprocal space of $\mathrm{PC}$ transform in similar way as the lattice vectors, or any point in the real space, do [29]. Therefore, points in the reciprocal space of $\mathrm{SC}\left(k_{x}^{\prime}, k_{y}^{\prime}, k_{z}^{\prime}\right)$ are related with equivalent points in the reciprocal space of $\mathrm{PC}$ by an equivalent transformation given by Eq. (3) and the transformation matrix defined in Eq. (4):

$\left(k_{x}, k_{y}, k_{z}\right)=\left(k_{x}^{\prime}, k_{y}^{\prime}, k_{z}^{\prime}\right) \mathbf{P}$.

For a $2 \mathrm{D}$ hexagonal lattice, $k_{z}=k_{z}^{\prime}=0$ and $\mathbf{P}$ is defined through Eq. (5).

Finally, we can calculate the electronic states of commensurate 2D Moiré SCs with the new coordinates $\left(k_{x}^{\prime}, k_{y}^{\prime}, 0\right)$ based on periodic DFT, and thereafter, we can apply the unfolding described above to calculate $W_{k}(I)$, obtaining the SC projected band structures onto the original PC systems. Since we are performing DFT calculations with a non-orthogonal basis-set [30], we employ a Gram-Schmidt process [31] to orthogonalize the Kohn-Sham orbitals and carry out the unfolding process through the calculation of $W_{k}(I)$ via Eq. (2). We want to stress that our unfolding code reads the whole information of wavefunctions from the corresponding SIESTA file and, then, performs the $W_{k}(I)$ calculation. Beside with the linear scaling [30], SIESTA code has different advantages: the choice 
of the basis-set size, different subroutines for writting the wavefunctions file, and the setting of how many $\mathrm{k}$ points will be calculated. Putting all this together, our unfolding code is able to work efficiently for twisted systems with significant number of atoms [27] with respect to other unfolding DFT methods based on plane-waves [20, 21], being able to unfold the electronic states even from the 1st occupied band up to the $I$-th desired band and improving the resolution of projected band structure easily by the number of $\mathrm{k}$ points along the paths.

\section{Models}

In order to show the validity and main characteristics of the present unfolding method, we study three structures: a conventional SC of monolayer G, a nonconventional SC of monolayer $\mathrm{G}$, and a twisted bilayer G-WS $\mathrm{W}_{2}$ heterostructure. First, let us describe the cases of monolayer G. Lattice vectors of the PC are $a=a_{G}(1,0,0), \quad b=a_{G}(-1 / 2, \sqrt{3} / 2,0)$ and $c=$ $c(0,0,1)$, where $a_{G}=2.44 \AA$ is the lattice parameter optimized through DFT calculations, which is in very good agreement with other reports [5, 32], and $c$ is the vacuum space imposed for the SC (see the computational details in Section 4). Conventional and non-conventional $\mathrm{SC}$ of monolayer $\mathrm{G}$ have a $3 \times 3$ and $\sqrt{13} \times \sqrt{13}$ periodicity, respectively. They are constructed using Eq. (3) and Eq. (5) with $m=3$ and $n=0$ for $\mathrm{G}(3 \times 3)$ with $\mathcal{N}=9$, and $m=3$ and $n=1$ for $\mathrm{G}(\sqrt{13} \times \sqrt{13})$ with $\mathcal{N}=13$. Inset of Figure 1 (a) and (d) show both SC. Hence, the total number of atoms, $N_{T}$, defined as $2 \mathcal{N}$, are 18 and 26 atoms, respectively. In general, the rotation angle, $\theta$, between two hexagonal layers is defined as:

$\cos \theta=\frac{m p+n q+(m q+n p) / 2}{\sqrt{m^{2}+n^{2}+m n} \times \sqrt{p^{2}+q^{2}+p q}}$,

where $m$ and $n$ indexes correspond with one of the rotated layer, whereas $p$ and $q$ correspond with the other one. Taking one of the layers as a conventional SC, $p \neq 0$ and $q=0$ and Eq. (7) simplifies:

$\cos \theta=\frac{m p+(n p) / 2}{p \times \sqrt{m^{2}+n^{2}+m n}}$,

giving $\theta=13.90^{\circ}$.

Now, let us describe the case of twisted G-WS $\mathrm{WS}_{2}$ bilayer. $\mathrm{PCs}$ of $\mathrm{G}$ and $\mathrm{WS}_{2}$ have hexagonal symmetry; hence, both PCs exhibit the same lattice vectors but different lattice parameter [see Figure 2(a) and (b)]. Letting an optimization of the lattice parameter of $\mathrm{WS}_{2}$, we obtain $a_{W S_{2}}=3.16 \AA$, which is in very good agreement with other reports $[33,34]$. Since the $\mathrm{PCs}$ of $\mathrm{G}$ and $\mathrm{WS}_{2}$ have different lattice parameters, the construction of a periodic vertically stacked $\mathrm{SC}$ of $\mathrm{G}-\mathrm{WS}_{2}$ is an additional problem. To overcome this problem, it is necessary to find $\mathrm{SC}$ of $\mathrm{G}$ and $\mathrm{WS}_{2}$ with similar lattice parameters; then, one of the layers is fixed and the other one is enlarged or reduced to assure commensurability. In our case, we fixed $\mathrm{WS}_{2}$ as the substrate and $\mathrm{G}$ is slightly enlarged or shrunk to match both lattice parameters of SCs. Thus, the in-plane strain $(\Delta)$ imposed in $\mathrm{G}$ and associated to commensurability condition is an intrinsic property, defined as [35]:

$$
\Delta=\frac{a_{G} \sqrt{m^{2}+n^{2}+m n}-a_{W S_{2}} \sqrt{p^{2}+q^{2}+p q}}{a_{W S_{2}} \sqrt{p^{2}+q^{2}+p q}},
$$

where $m$ and $n$ indexes correspond to $\mathrm{G}$, while $p$ and $q$ do to $\mathrm{WS}_{2}$, obeying the following constrains: $m \geq n$ and $p \geq q$ and $|\Delta| \leq 1.5 \%$ [35]. Then, we employ the matrix transformation described in Eq. (5) with $m=2, n=2$ for $\mathrm{G}$, and $p=2, q=1$ for $\mathrm{WS}_{2}$, plus the lattice vectors of $\mathrm{G}$ and $\mathrm{WS}_{2}$ to generate the periodic $\mathrm{SC}$ of $\mathrm{G}-\mathrm{WS}_{2}$, shown in Figure 2(c). For these indexes, $\Delta=-1.09 \%$, where the minus sign means compression of the $\mathrm{G}$ layer, the twisted-angle $\theta=10.9^{\circ}$, the lattice parameter of the $\mathrm{SC}$ is $8.34 \AA$, and the total atoms of the SC is 45 . Visualization of atomic models was performed using the VESTA program [36].

\section{Computational details}

DFT calculations are performed using the SIESTA code [30]. Exchange-correlation energy is described by local density approximation (LDA) with the Perdew-Zunger parametrization [37]. Norm-conserving pseudopotentials [38] in their scalar relativistic and non-local form [39] are employed for electron-ion interactions. The expansion of the valence electronic states is described by a LCAO with an optimized double- $\zeta$ polarized (DZP) basis-set plus diffuse orbitals $[40,41,42]$. If the basis-set uses strictly localized orbitals, it is necessary to include diffuse orbitals for a better description of interface and vacuum regions in slabs and surfaces [40]. The electronic density is sampled by a mesh-cutoff energy of $300 \mathrm{Ry}$ and converged up to $10^{-5}$. The Monkhorst-Pack scheme [43] is used for sampling the BZ with an optimal $25 \times 25 \times 1 k$-grid. Energy mesh-cutoff and $k$-grid convergence tests were performed leading to the above mentioned values as the optimal ones. A Fermi-Dirac smearing of $1 \mathrm{~K}$ is adopted to obtain convergence. The structural optimization is carried out within the conjugate gradient algorithm with a maximum value of $0.01 \mathrm{eV} / \AA$ for the interatomic forces, as well as $0.5 \mathrm{GPa}$ in the stress components. A vacuum space of $55 \AA$ is applied to avoid spurious interactions between periodic images along $\mathbf{c}$ direction. Finally, all calculations include a dipole correction, as it was suggested by Bengtsson [44], to cancel the artificial electric field 

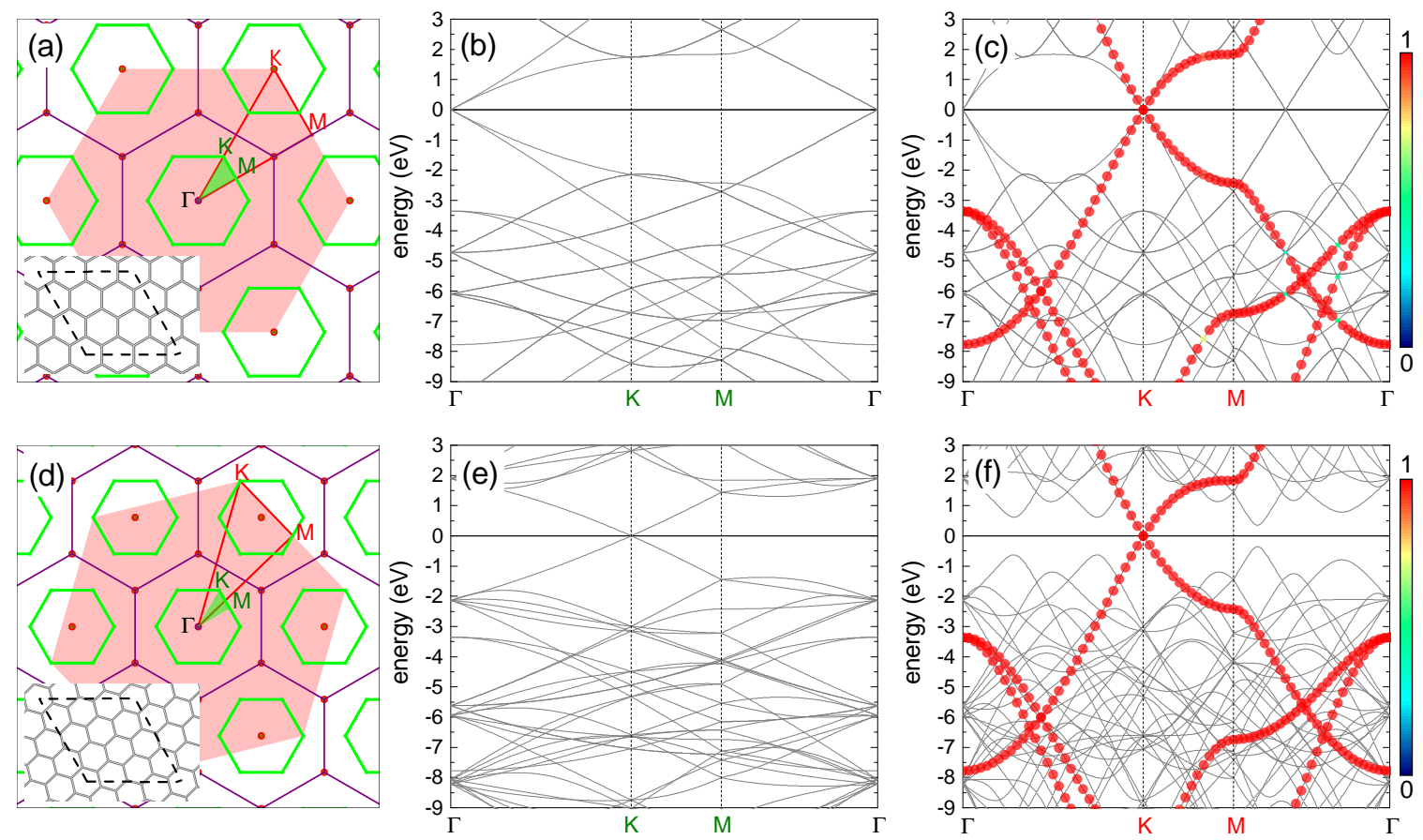

Figure 1. Top row: (a) reciprocal space of $\mathrm{G}(3 \times 3) \mathrm{SC}$. The green (shaded-red) hexagons denotes the first Brillouin zone of G SC (PC). The irreducible Brillouin zone of SC (PC) is enclosed by the filled-green (red) triangle. High symmetry points are coloured in green (red) for $\mathrm{SC}(\mathrm{PC})$. The inset shows the stick representation of $\mathrm{G}(3 \times 3) \mathrm{SC}$ layer in real space and the dashed-black lines delimit the size of SC. (b) calculated band structure for $\mathrm{G}(3 \times 3)$ SC. The plot (c) shows the SC electronic states (gray background) calculated using the red paths in (a) while, after the projection, those SC electronic states with the same symmetry of that reference $\mathrm{PC}$ are plotted with circles of different size and color, which are proportional to $W_{k}(I)$. The bottom row depicts the characteristics for $\mathrm{G}(\sqrt{13} \times \sqrt{13}) \mathrm{SC}$ as the top row. The number of k-points scanned along $\Gamma \rightarrow \mathrm{K}, \mathrm{K} \rightarrow \mathrm{M}$ and $\mathrm{M} \rightarrow \Gamma$ were 30 , 15 and 30 , respectively. The Fermi level is set to $0 \mathrm{eV}$.

(a)

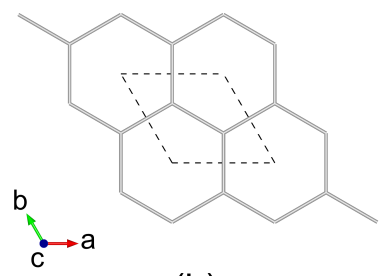

(b)

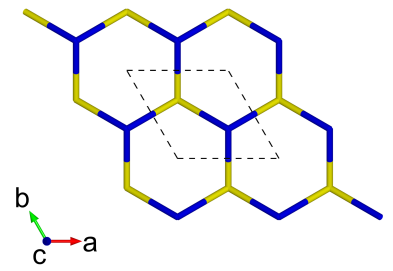

(c)

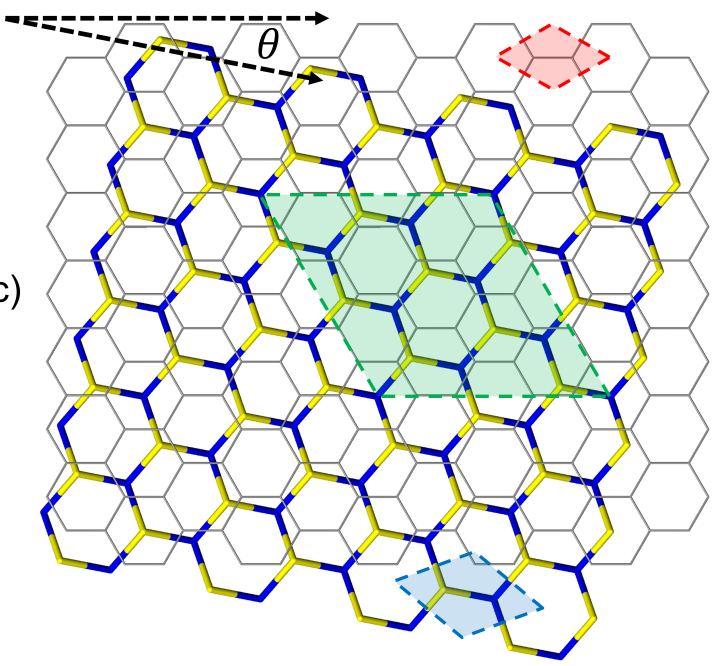

Figure 2. Top view of stick representation of (a) G PC, (b) $\mathrm{WS}_{2} \mathrm{PC}$ and (c) G/WS 2 SC. The PCs in (a) and (b) have the same orientation and indicated by dashed lines. In (c), the $\mathrm{G} / \mathrm{WS}_{2} \mathrm{SC}$ is highlighted by the green area, while $\mathrm{G}$ and $\mathrm{WS}_{2} \mathrm{PCs}$ are in red and blue areas, respectively. $\theta$ is the angle between the armchair edges of both layers. Color code: gray, yellow, and blue sticks represent $\mathrm{C}, \mathrm{S}$ and $\mathrm{W}$ elements, respectively. 
induced by the vertical asymmetry of the SC and the periodic boundary conditions.

\section{Results}

As a first proof of our unfolding code, we study the electronic structure of an ideal $\mathrm{G}(3 \times 3)$ conventional layer. The reciprocal space, the first $\mathrm{BZ}$ and the irreducible BZ of the $\mathrm{SC}$ and $\mathrm{PC}$ are shown in Figure 1 (a). Also, the inset presents the $\mathrm{G}(3 \times 3)$ conventional layer. Figure 1(b) shows the electronic band structure of the SC. The typical linear dispersion of electronic states of the PC at the Fermi level around the $\mathrm{K}$ point is also present in $\mathrm{SC}$ but around the $\Gamma$ point. Due to the folding process, many electronic states are merged into the small SC reciprocal space [the green area in Figure 1(a)]. The irreducible BZ of the PC has the fractional coordinates $\mathrm{K}=(1 / 3,1 / 3,0)$ and $\mathrm{M}=(1 / 2,0,0)$ in terms of the corresponding reciprocal lattice vectors of $\mathrm{PC}$. After the transformation with Eqs. (6) and (4), the new fractional coordinates of high-symmetry points are $\mathrm{K}=(1,1,0)$ and $\mathrm{M}=$ $(3 / 2,0,0)$ but in terms of the reciprocal lattice vectors of SC. With this transformation, the highly symmetric points in the reciprocal space of $\mathrm{SC}$ can be easily identified in the reciprocal space of PC [see Figure 1(a)]. When we unfold the electronic states of SC calculated throughout the red path of $\mathrm{G}$ [gray band structure in Figure 1(c)] onto the PC of G, the fully band structure of the ideal $\mathrm{PC}$ is recovered, as it is shown by red circles in Figure 1(c), which means $W_{k}(I)=1$. Some unfolded wavefunctions in Figure $1(\mathrm{c})$ have $W_{k}(I) \neq 1$ along $\mathrm{M}-\Gamma$ within -7.5 and $4.5 \mathrm{eV}$ range, given by green dots and the yellow dot along $\mathrm{K}-\mathrm{M}$ at $-7.5 \mathrm{eV}$, because the wavefunctions are degenerated due to scanned k-points match with Bragg diffractions. However the total summation of $W_{k}(I) \mathrm{s}$ is $\simeq 1$. This characteristic of our unfolding process allows seeing two main aspects in comparison with previous unfolding methods with DFT [16, 17, 18, 19, 20, 21] or tight-binding calculations $[22,23,24]$ : first, we can determine a relationship between the reciprocal space and possible Bragg diffractions in the band structure; and, second, electronic degeneracy and/or hybridization can be quantified via $W_{k}(I)$. These capabilities of our unfolding code are owing to the basis-set used and the normalization process and they will be discussed with more details later for the $\mathrm{G} / \mathrm{WS}_{2}$ heterostructure.

We performed the same analysis for $\sqrt{13} \times \sqrt{13}$ G layer with $\theta=16.1^{\circ}$. Figure $1(\mathrm{~d})$ sketches the reciprocal space, the first and the irreducible $\mathrm{BZ}$ of $\mathrm{SC}$ with the same orientation as those in Figure 1(a). The inset shows the corresponding SC layer. Again, the typical linear dispersion of electronic states of
$\mathrm{SC}$ at the Fermi level around the $\mathrm{K}$ point is also present. Comparing Figure 1(e) and 1(b), there are more electronic bands (gray lines as background) in the former at a glance. Nevertheless, when the unfolding process is applied using the new fractional coordinates $\mathrm{K}=(2 / 3,5 / 3,0)$ and $\mathrm{M}=(3 / 2,1 / 2,0)$ of $\mathrm{PC}$, the fully band structure of $\mathrm{PC}$ is again fully recovered, as in Figure 1(f) with $W_{k}(I)=1$. An analysis of unfolded bands of Figure 1(f) reveals the absence of states with $W_{k}(I) \neq 1$, since the coordinates $\left(k_{x}, k_{y}\right)$ of scanned kpoints of $\mathrm{SC}$ are not the same positions as Bragg points or lines in reciprocal space. Therefore, our unfolding code works for conventional hexagonal SCs, and nonconventional ones as well.

Now we turn to a more complex system: a heterostructure built up by of two twisted nonconventional SCs. In Figure 2(c), the 2D commensurable heterostructure composed of $2 \sqrt{3} \times 2 \sqrt{3} \mathrm{G}$ and $\sqrt{7} \times \sqrt{7} \mathrm{WS}_{2}$ with an interlayer rotation angle $\theta=10.9^{\circ}$ is depicted. Hence, the number of times that the $\mathrm{PC}$ is repeated on the $\mathrm{SC}, \mathcal{N}$ in Eq. (2), or equivalently the determinant of $\mathbf{P}$ is 12 for $\mathbf{G}$, and 7 for $\mathrm{WS}_{2}$. The optimized average interlayer distance, $d$, defined as the average distance between carbon atoms of $\mathrm{G}$ and upper sulfur atoms of $\mathrm{WS}_{2}$ is $d=3.28 \AA$, in good agreement with previous reports $[45,46]$. Average distances of carbon-carbon $(\mathrm{C}-\mathrm{C})$, tungsten-sulfur $(\mathrm{W}-\mathrm{S})$ and up and down sulfur atoms $\left(\mathrm{S}_{u}-\mathrm{S}_{d}\right)$ are 1.41, 2.41 and $3.16 \AA$, respectively. The irreducible BZ of $\mathrm{PC}$ of both $\mathrm{WS}_{2}$ and $\mathrm{G}$ has the same fractional coordinates. Once the transformation is done using Eqs. (6) and (5) on the high-symmetry points, the new fractional positions are $\mathrm{K}=(0,2,0)$ and $\mathrm{M}=(1,1,0)$ for $\mathrm{G}$, and $\mathrm{K}=(1 / 3,4 / 3,0)$ and $\mathrm{M}=(1,1 / 2,0)$ for $\mathrm{WS}_{2}$. Multiplying these new fractional positions by the reciprocal lattice vectors of SC generate the coordinate points of the twisted irreducible BZ of PCs in Figure 3(a), where the red labels identify corresponding $\mathrm{BZ}$ of $\mathrm{G}$, while blue labels do with $\mathrm{WS}_{2}$.

Using the fractional coordinates $\mathrm{K}=(1 / 3,1 / 3,0)$ and $\mathrm{M}=(1 / 2,0,0)$ of the $\mathrm{SC}$, we calculated the electronic band structure of the heterostructure $\mathrm{G} / \mathrm{WS}_{2}$ scanning the $\Gamma-\mathrm{K}-\mathrm{M}-\Gamma$ path, as it is shown in Figure 3(b). Close to the Fermi level, the typical linear energy dispersion associated to the Dirac cones of $G$ is observed at the $\Gamma$ point. The preserving of the linear dispersion in $2 \mathrm{D}$ van der Waals heterostructures has been reported in other works [32, 47, 35, 48, 49] by means of ab initio calculations. Furthermore, close to the Fermi level the degeneracy is broken at the $\Gamma-\mathrm{M}$ path, while it is preserved at the $\Gamma-\mathrm{K}$ path. For energies lower than $-1 \mathrm{eV}$ and greater than +1 $\mathrm{eV}$ there are many electronic states associated to $\mathrm{G}$ and $\mathrm{WS}_{2}$ as well, which means that the electronic states of $G$ around the Fermi level fall inside the 

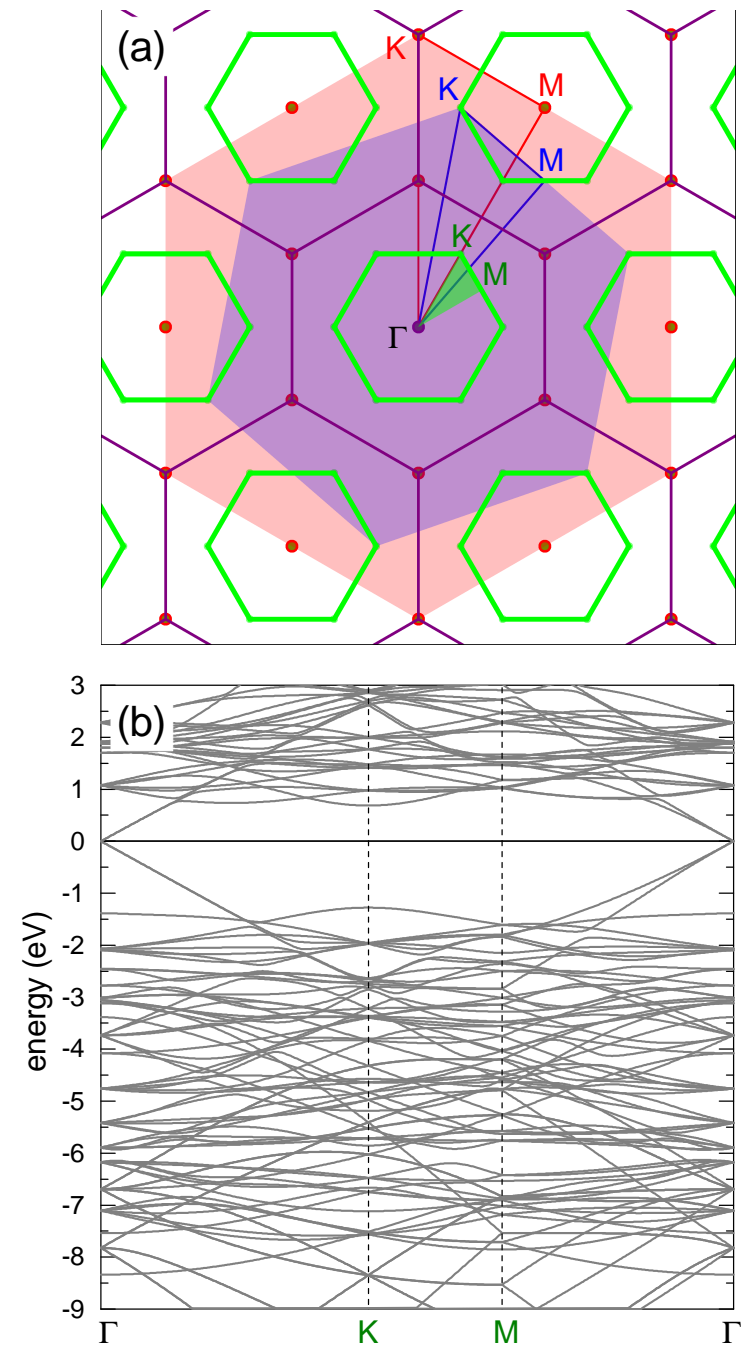

Figure 3. (a) Reciprocal space of the $\mathrm{G} / \mathrm{WS}_{2}$ system. The purple (joined by the red points), green (green points) hexagons represent the reciprocal space and the first BZ. The irreducible $\mathrm{BZ}$ of $\mathrm{SC}$ is represented by a shaded green area. The red and blue hexagons represent the first $\mathrm{BZ}$ of $\mathrm{PC}$ of $\mathrm{G}$ and $\mathrm{WS}_{2}$, respectively, and the red (blue) triangle denotes the path of the irreducible BZ for $\mathrm{G}\left(\mathrm{WS}_{2}\right)$. $\mathrm{K}$ and $\mathrm{M}$ labels are in green, red and blue for the $\mathrm{G} / \mathrm{WS}_{2}$, and both $\mathrm{PC}$ of $\mathrm{G}$ and $\mathrm{WS}_{2}$, respectively, while the $\Gamma$-point (in black) is a common reference among the three systems. (b) Electronic band structure of $\mathrm{G} / \mathrm{WS}_{2}$ with the Fermi level set to $0 \mathrm{eV}$.

band gap of $\mathrm{WS}_{2}$, confirming that the full system is a semimetal. From a simple analysis of Figure 3(b), it is quite difficult to extract more physical information. Nonetheless, applying our unfolding method to the electronic band structure of $\mathrm{G} / \mathrm{WS}_{2}$ onto each $\mathrm{PC}$, it is possible to distinguish the physical consequences of mutual interactions among layers.

Let us apply our unfolding code to the $\mathrm{G} / \mathrm{WS}_{2}$ structure to project the electronic states to both PC using the corresponding $\Gamma-\mathrm{K}-\mathrm{M}-\Gamma$ path for $\mathrm{G}$ and $\mathrm{WS}_{2}$ [see Figure 3(a)]. Once the corresponding $W_{k}(I)$ values are obtained, the effective electronic bands (EEBs) for $\mathrm{G}$ and $\mathrm{WS}_{2}$ are plotted, as it is shown in Figure 4(a) and 4(b), respectively. In general, EEBs show large difference and discontinuities with respect to the electronic band structure of pristine PC of $\mathrm{G}$ $[50,51]$ and $\mathrm{WS}_{2}[52,33,34]$ as a consequence of $W_{k}(I)$ is not exactly equal to 1 in every projected band. Such deviations can be easily observed comparing EEB of $\mathrm{G}$ in Figure 5(a) with the band structure of pristine $\mathrm{PC}$ of $\mathrm{G}$ in Figure 1(c) and 1(f). This means that the symmetry of each layer reconstruction is not equal to the original symmetry of pristine PC. In particular, the translation symmetry is broken in this $2 \mathrm{D} \mathrm{G} / \mathrm{WS}_{2}$ heterostructure due to interlayer interaction.

In the case of $\mathrm{G}$, the unfolding process shows that the Dirac cone around the Fermi level remains unchanged but it is now located at the $\mathrm{K}$ point of PC instead of $\Gamma$ of SC. Furthermore, it falls inside of the $\mathrm{WS}_{2}$ bandgap. At the Fermi level, there is no bandgap associated to $\mathrm{G}$ bands despite the applied symmetrical strain, $\Delta$, which is in agreement with other theoretical results for pristine $G$ undergoes a small symmetrical strain distribution [53]. On the other hand, we detected a change in Fermi velocity $\left(v_{F}\right)$ in $\mathrm{G}$ by the strain. A slight increase of $1.54 \%$ was measured, taking as reference the unfolded band structure of G SC in Figure 1(c). The previous $v_{F}$ value was roughly estimated only for carriers in the valence band. Our results are in good agreement with other experimental and theoretical results $[54,53]$ with potential applications in strain engineering and flexible electronics. For energies below $-3 \mathrm{eV}$ in figure 5(a), there are two bands composed of mixed $\mathrm{C}\left(2 s, 2 p_{x}, 2 p_{y}\right)$ orbitals giving a $s p^{2}$ hybridization. These bands are non-perturbed showing a $W_{k}(I) \simeq 1$ along the full $k$-path. Thus, since we performed DFT calculations with a localized basis-set, we can extract partial EEBs taking into account only specific orbitals or atoms of the SC [27]. This result reflects the usefulness of the present unfolding code based on atomic orbitals and normalized wavefunctions for studying of any perturbed periodic system. At the energies plotted in Figure 5 , only bands associated with $\mathrm{C}\left(2 p_{z}\right)$ orbitals changes drastically. It is observed a splitting bands near to $\Gamma$ point at energies around $-8 \mathrm{eV}$, which is similar to the band structure of graphite [55]. Also, different minigaps in the valence bands are observed. Such minigaps observed at low energies are in fair agreement with experimental results of $\mathrm{G}$ adsorbed on $\mathrm{MoS}_{2}[56,57]$, as well as other theoretical calculations $[26,25]$ with $\theta=0^{\circ}$. We will discuss more about the formation of minigaps latter. Another advantage of the unfolding method for studying these systems in comparison, for instance, with ARPES [15] technique is that, we can see what happens with the unoccupied 
states as well. In this case, in Figure 4(a) there is not an equal relation of $W_{k}(I)$ for each wave function (I) and $k$-point, which means there is a particle-hole asymmetry and, as result of $\mathrm{WS}_{2}$ interaction, the mobility of holes and electrons would behave different in $\mathrm{G}[24,58]$.

Now, let us discuss the unfolding process projecting the electronic states of G/WS2 to the PC of $\mathrm{WS}_{2}$. The direct-transition electronic band gap located at the $\mathrm{K}$ point of $\mathrm{PC}$ is preserved, which means that, under our methodology, the vertical stacking of $\mathrm{G}$ with $\mathrm{WS}_{2}$ does not change the electronic properties of $\mathrm{WS}_{2}$ near the Fermi energy. In the $\Gamma-\mathrm{K}$ path and around $-1.5 \mathrm{eV}$, a small discontinuity is observed in the last occupied valence band (second unnocupied band), green arrows in Figure 4(b). Going down in energy, all electronic bands of $\mathrm{WS}_{2}$ change from $-2 \mathrm{eV}$ to $-6 \mathrm{eV}$, which means that $\mathrm{W}(d)$ and $\mathrm{S}(p)$ orbitals modify their occupation just by $\mathrm{G}$ adsorption. Another interesting result obtained through the unfolding process is the energy splitting about $\sim 1 \mathrm{eV}$ of the band at $\Gamma-\mathrm{K}$ and $\mathrm{M}-\Gamma$ path from $-7.5 \mathrm{eV}$ to $-8.5 \mathrm{eV}$ [see Figure $4(\mathrm{~b})$ ], also observed in the PC of G [see Figure 4(a)], which means an interlayer interaction repulsion between the $\mathrm{C}\left(2 p_{z}\right)$ orbitals of $\mathrm{G}$ and $\mathrm{S}(3 p)$ and $\mathrm{W}(5 d)$ orbitals of $\mathrm{WS}_{2}$. Furthermore, it is noticeable that there is a complementary relationship between the two EEBs of $\mathrm{G}$ and $\mathrm{WS}_{2}$ around the $\Gamma$ point for energies from $6 \mathrm{eV}$ to $-8.5 \mathrm{eV} . W_{k}(I)$ of $\mathrm{G}$ bands [see Figure $4(\mathrm{a})$ ] complements the $W_{k}(I)$ of $\mathrm{WS}_{2}$ [see Figure 4(b)], even when the scanned paths are a bit different near the $\Gamma$ point. This complementary characteristic of $W_{k}(I)$ between two monolayers is another advantage of our unfolding process, in comparison with other unfolding methods based on Green's functions for periodic twisted bilayers $[24,26]$.

Regarding the electronic minigaps observed in EEBs of $\mathrm{G}$, it is worth pointing out that their number, size and position in this $\mathrm{G} / \mathrm{WS}_{2}$ heterostructure with $\theta=10.9^{\circ}$ is greater than other theoretical reports when $\theta=0^{\circ}[26,25]$. This means that $\theta$ has a strong influence on the electronic properties in $2 \mathrm{D}$ heterostructures, as in the case of $\mathrm{G}$ bilayer $[5,6,7,8]$. We have identified two kind of electronic interactions. One is related with the electronic gap generation with a direct transition. The other one seems an electronic resonance (or discontinuity) between outof-plane orbitals of each monolayer. It is possible to identify both behaviors in the EEBs of G. In Figure 4(a), the minigaps are observed along the $\Gamma-\mathrm{K}$ and $\mathrm{M}$ $\Gamma$ paths and indicated by white arrows, while electronic resonances are detected in the K-M path indicated by green arrows near the $\mathrm{M}$ point. Based on the reciprocal space sketched in Figure 3, we can say that the gaps located in $1 / 2$ and 0.7 starting from $\Gamma \rightarrow \mathrm{K}$ path and those located at 0.22 and near to $1 / 3$ and $1 / 2$ starting from $M \rightarrow \Gamma$ path are Bragg diffractions. The rest of minigaps have a different physical origin. The discontinuity in K-M path looks like an electronic resonance showing coupling between monolayers. The Bragg diffraction in $\mathrm{WS}_{2}$ located at $0.6(1 / 3) \Gamma \rightarrow \mathrm{K}$ $(\mathrm{M} \rightarrow \Gamma)$ path shows minigaps around these points for energies from $-5.5 \mathrm{eV}$ to $-7.5 \mathrm{eV}$. Also, other minigaps are observed near the $\mathrm{K}$ (on left-side) and $\mathrm{M}$ points (on right-side) with energy $-3.5 \mathrm{eV}$ indicated by arrows. A discontinuity is observed in $0.6 \Gamma \rightarrow \mathrm{K}$ path in $\sim 2.75$ $\mathrm{eV}$ below the Fermi level. Finally, the number of Bragg diffractions in $\mathrm{G}$ is greater than $\mathrm{WS}_{2}$ by the size of $\mathrm{BZs}$ of PCs. The origin of all different interactions will be discussed in a future work.

We want to stress that, strictly speaking if both layers do not interact between them, $W_{k}(I)$ must be equal to 1 as in Figures 1(c) and 1(f). To corroborate this, we analyze the $\mathrm{G} / \mathrm{WS}_{2}$ heterostructure with the interlayer electronic interactions almost zero. This condition is possible putting both layers separated by an interlayer distance larger than the equilibrium one $d=3.28 \AA$. Hence, using the optimized $\mathrm{G} / \mathrm{WS}_{2}$ structure, we impose an interlayer distance of 5.29 $\AA$ and, from a single-point DFT calculation, the electronic states of the SC are obtained. Then, we applied our unfolding code to project the electronic states to both PC using the corresponding $\Gamma-\mathrm{K}-\mathrm{M}-\Gamma$ path for $\mathrm{G}$ and $\mathrm{WS}_{2}$, denoted in Figure 3(a) by red and blue labels, respectively. Once the corresponding $W_{k}(I)$ values are obtained, the EEBs are plotted. Figure 5(a) shows with red and blue points the EEB of $\mathrm{G}$ and $\mathrm{WS}_{2}$ projected onto the $\Gamma-\mathrm{K}-\mathrm{M}-\Gamma$ path for G, while 5(b) shows the corresponding EEB projected onto the $\Gamma-\mathrm{K}-\mathrm{M}-\Gamma$ path for $\mathrm{WS}_{2}$. Analyzing the EEBs of $\mathrm{G}$ and $\mathrm{WS}_{2}$ in Figure 5(a) and 5(b), respectively, they are almost entirely recovered since $W_{k}(I) \simeq 1$ in the whole energy range and the scanned $\mathrm{k}$ path as well. This is an outstanding theoretical proof which shows that the strong interlayer interaction, given by $\mathrm{vdW}$ forces, leads to hybridization and the emerging of minigaps discussed above. However, some KohnSham orbitals show $W_{k}(I)<1$, which is associated to interlayer distortions originated by the structural optimization carried out at $d=3.28 \AA$. Comparing both Figures 5(a) and (b), the EEBs of $\mathrm{G}$ are quite different as well as corresponding of $\mathrm{WS}_{2}$. Such differences are originated because in Figure 5(a) the states of $\mathrm{WS}_{2}$ are projected onto the $\mathrm{BZ}$ of $\mathrm{G}$, while in Figure 5(b) the states of $\mathrm{G}$ are projected onto the BZ of $\mathrm{WS}_{2}$. So, a wrong choice of the $\mathrm{k}$ path could induce a misunderstanding or even a bad interpretation of the mutual influence between layers. Additionally, in every plot in Figure 5 we can observe only the electronic states from the reference PCs without the presence of 

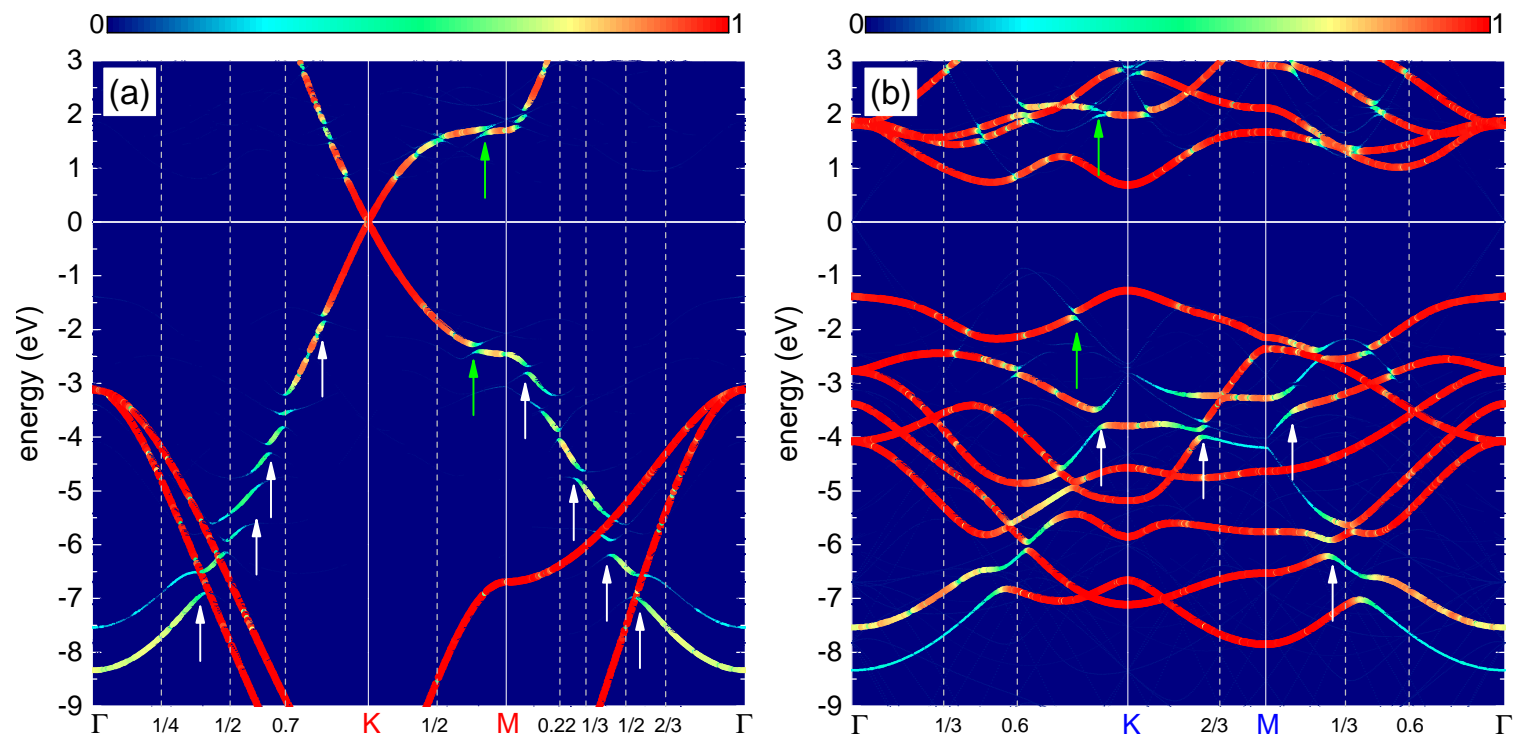

Figure 4. Calculated EEBs for (a) G and (b) $\mathrm{WS}_{2}$ PCs with an interlayer distance of $3.28 \AA$. The vertical dashed lines represent Bragg points and lines in the reciprocal space. The fractional values along the paths mean the position at which diffractions occur. Minigaps with an origin different from Bragg reflections are indicated by white arrows, while resonances states are indicated by green arrows. The number of k-points scanned along $\Gamma \rightarrow \mathrm{K}, \mathrm{K} \rightarrow \mathrm{M}$ and $\mathrm{M} \rightarrow \Gamma$ were 200, 100 and 200 , respectively. The $W_{k}(I)$ is proportional to symbol size and color. The Fermi level is set to $0 \mathrm{eV}$.

additional bands or the so-called ghost bands [26]. This is another advantage of the present unfolding method based on atomic orbitals and DFT. Comparing the EEB of strained $G$ in Figure 5(a) with ideal G bands in Figure 1(c) around $-8 \mathrm{eV}$ and close to $\Gamma$ point, a shift energy of the electronic state are observed, which is directly related with the strain condition of $\mathrm{G}$ monolayer. Meanwhile $\mathrm{WS}_{2}$ is a direct bandgap semiconductor at the $\mathrm{K}$ point in its $\mathrm{PC}$, see blue circles in Figure 5(b).

\section{Conclusions}

In summary, total energy density functional theory calculations in combination with an extended unfolding band structure approach have been applied to study conventional and non-conventional graphene supercells, and twisted bilayer of non-conventional graphene and tungsten disulfide supercells. The unfolding method works for periodically twisted systems, showing that, the full band structure of the primitive cells are recovered if ideal supercells of graphene are studied. Furthermore, we addressed the topic of interaction by proximity effects in a system of graphene and tungsten disulfide with double-periodicity. Several advantages of developed method have been presented and the physics of interlayer interactions is easier to explain. When graphene is adsorbed on tungsten disulfide, many electronic interactions arise between monolayers when the interlayer distance is reduced until its optimal value. The results reveal a strong modulation of the electronic structure of graphene by tungsten disulfide due to the relatively weak van der Waals interactions. In graphene, the main contributions are by the orbital normal to the monolayer planes, while the in-plane orbitals form strong bonds without any perturbation. However, around the Fermi level the Dirac cone remains almost unaffected with small changes in the Fermi velocity of the carriers assisted by symmetric lattice strain. The electronic properties of tungsten disulfide are preserved near the Fermi energy, mainly the direct band gap at the same point. The nature of minigaps comes from Bragg conditions in the supercell and the interlayer coupling between graphene and tungsten disulfide monolayers.

\subsection{Acknowledgments}

Authors acknowledge the partial support from DGAPA-UNAM project PAPIIT IN109618 and CONACyT project A1-S-39242. F.S.O. acknowledges to DGAPA- UNAM for postdoctoral fellowship. The numerical calculations were carried out in clusters Baktum and Flatland at the Institute of Physics (UNAM).

\section{References}

[1] Geim A K and Grigorieva I V 2013 Nature 499419

[2] Bhimanapati G R, Lin Z, Meunier V, Jung Y, Cha J, Das S, Xiao D, Son Y, Strano M S, Cooper V R et al. 2015 ACS nano 9 11509-11539 

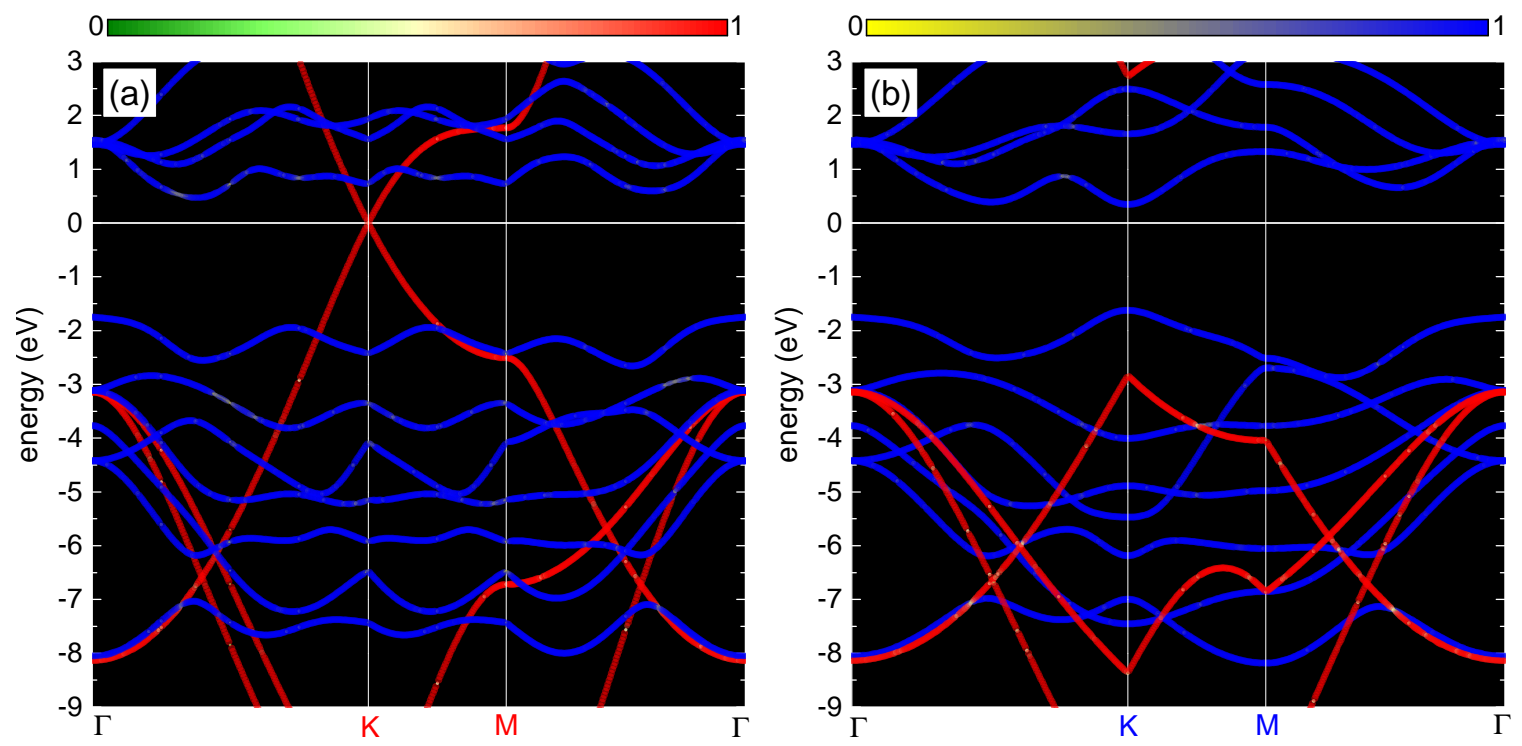

Figure 5. Calculated EFBSs for (a) G and (b) $\mathrm{WS}_{2}$ PCs. An interlayer distance of $5.29 \AA$ reflects weak electronic interactions between $\mathrm{G}$ and $\mathrm{WS}_{2}$. The projection of $\mathrm{G} / \mathrm{WS}_{2} \mathrm{SC}$ states onto the $\mathrm{WS}_{2}(\mathrm{G}) \mathrm{PC}$ using the k-path of $\mathrm{G}(\mathrm{WS} 2)$ is plotted on left (right) panel with blue (red) circles. The number of k-points scanned along $\Gamma \rightarrow \mathrm{K}, \mathrm{K} \rightarrow \mathrm{M}$ and $\mathrm{M} \rightarrow \Gamma$ were 200 , 100 and 200 , respectively. The $W_{k}(I)$ is proportional to symbol size and color. The upper color bar from green to red (yellow to blue) is for $W_{k}(I)$ of $\mathrm{G}\left(\mathrm{WS}_{2}\right)$. The Fermi level is set to $0 \mathrm{eV}$.

[3] Lu C C, Lin Y C, Liu Z, Yeh C H, Suenaga K and Chiu P W 2013 ACS nano 7 2587-2594

[4] Dean C R, Wang L, Maher P, Forsythe C, Ghahari F, Gao Y, Katoch J, Ishigami M, Moon P, Koshino M et al. 2013 Nature 497598

[5] Kim C J, Sánchez-Castillo A, Ziegler Z, Ogawa Y, Noguez C and Park J 2016 Nat. Nanotechnol. 11520

[6] Rickhaus P, Wallbank J, Slizovskiy S, Pisoni R, Overweg H, Lee Y, Eich M, Liu M H, Watanabe K, Taniguchi T, Ihn T and Ensslin K 2018 Nano Lett. 18 6725-6730

[7] Cao Y, Fatemi V, Fang S, Watanabe K, Taniguchi T, Kaxiras E and Jarillo-Herrero P 2018 Nature 55643

[8] Cao Y, Fatemi V, Demir A, Fang S, Tomarken S L, Luo J Y, Sanchez-Yamagishi J D, Watanabe K, Taniguchi T, Kaxiras E et al. 2018 Nature 55680

[9] Roy K, Padmanabhan M, Goswami S, Sai T P, Ramalingam G, Raghavan S and Ghosh A 2013 Nat. Nanotechnol. 8 826

[10] Zhang W, Chuu C P, Huang J K, Chen C H, Tsai M L, Chang Y H, Liang C T, Chen Y Z, Chueh Y L, He J H et al. 2014 Sci. Rep. 43826

[11] Georgiou T, Jalil R, Belle B D, Britnell L, Gorbachev R V, Morozov S V, Kim Y J, Gholinia A, Haigh S J, Makarovsky O et al. 2013 Nat. Nanotechnol. 8100

[12] Yu W J, Li Z, Zhou H, Chen Y, Wang Y, Huang Y and Duan X 2013 Nat. Mater. 12246

[13] Wang Z, Ki D K, Chen H, Berger H, MacDonald A H and Morpurgo A F 2015 Nat. Commun. 68339

[14] Ashcroft N W and Mermin N D 1976 Solid state physics (New York: Holt, Rinehart and Winston)

[15] Yang H, Liang A, Chen C, Zhang C, Schroeter N B and Chen Y 2018 Nat. Rev. Mater. 3 341-343

[16] Ku W, Berlijn T and Lee C C 2010 Phys. Rev. Lett. 104(21) 216401

[17] Popescu V and Zunger A 2010 Phys. Rev. Lett. 104(23) 236403

[18] Popescu V and Zunger A 2012 Phys. Rev. B 85(8) 085201

[19] Lee C C, Yamada-Takamura Y and Ozaki T 2013 J. Phys.:
Condens. Matter 25345501

[20] Medeiros P V, Stafström S and Björk J 2014 Phys. Rev. B 89041407

[21] Medeiros P V, Tsirkin S S, Stafström S and Björk J 2015 Phys. Rev. B 91041116

[22] Boykin T B and Klimeck G 2005 Phys. Rev. B 71(11) 115215

[23] Boykin T B, Kharche N, Klimeck G and Korkusinski M 2007 J. Phys.: Condens. Matter 19036203

[24] Nishi H, Matsushita Y i and Oshiyama A 2017 Phys. Rev. $B$ 95(8) 085420

[25] Le N B, Huan T D and Woods L M 2016 ACS Appl. Mater. Interfaces 8 6286-6292

[26] Matsushita Y i, Nishi H, Iwata J i, Kosugi T and Oshiyama A 2018 Phys. Rev. Materials 2 010801(R)

[27] Deretzis I, Calogero G, Angilella G and La Magna A 2014 EPL 10727006

[28] Haverkort M, Elfimov I and Sawatzky G 2011 arXiv preprint arXiv:1109.4036

[29] Arnold H 2006 International Tables for Crystallography. Vol. A, Chapter 5.1 (Netherlands: Springer)

[30] Soler J M, Artacho E, Gale J D, García A, Junquera J, Ordejón P and Sánchez-Portal D 2002 J. Phys.: Condens. Matter 142745

[31] Press W H, Teukolsky S A, Vetterling W T and Flannery B P 1992 Numerical recipes in Fortran 77, vol. $12 d$ ed. (Cambridge: Cambridge Univ. Press)

[32] Wang Z, Chen Q and Wang J 2015 J. Phys. Chem. C 119 $4752-4758$

[33] Klein A, Tiefenbacher S, Eyert V, Pettenkofer C and Jaegermann W 2001 Phys. Rev. B 64205416

[34] Yun W S, Han S, Hong S C, Kim I G and Lee J 2012 Phys. Rev. B 85033305

[35] Li L and Zhao M 2014 J. Phys. Chem. C 118 19129-19138

[36] Momma K and Izumi F 2011 J. Appl. Crystal. 44 12721276

[37] Perdew J P and Zunger A 1981 Phys. Rev. B 235048

[38] Hamann D, Schlüter M and Chiang C 1979 Phys. Rev. Lett. 
431494

[39] Kleinman L and Bylander D 1982 Phys. Rev. Lett. 481425

[40] García-Gil S, García A, Lorente N and Ordejón P 2009 Phys. Rev. B 79075441

[41] Hauschild A, Karki K, Cowie B, Rohlfing M, Tautz F and Sokolowski M 2005 Phys. Rev. Lett. 94036106

[42] Lee K, Yu J and Morikawa Y 2007 Phys. Rev. B 75045402

[43] Monkhorst H J and Pack J D 1976 Phys. Rev. B 135188

[44] Bengtsson L 1999 Phys. Rev. B 5912301

[45] Kaloni T, Kou L, Frauenheim T and Schwingenschlögl U 2014 Appl. Phys. Lett. 105233112

[46] Li S S and Zhang C W 2013 J. Appl. Phys. 114183709

[47] Di Felice D, Abad E, González C, Smogunov A and Dappe Y 2017 J. Phys. D: Appl. Phys. 50 17LT02

[48] Sa B, Sun Z and Wu B 2016 Nanoscale 8 1169-1178

[49] Wang Y and Ding Y 2015 Phys. Chem. Chem. Phys. 17 27769-27776

[50] Şahin H, Cahangirov S, Topsakal M, Bekaroglu E, Akturk E, Senger R T and Ciraci S 2009 Phys. Rev. B 80155453

[51] Boukhvalov D, Katsnelson M and Lichtenstein A 2008 Phys. Rev. B $\mathbf{7 7} 035427$

[52] Wang Q H, Kalantar-Zadeh K, Kis A, Coleman J N and Strano M S 2012 Nat. Nanotechnol. 7699

[53] Gui G, Li J and Zhong J 2008 Phys. Rev. B 78075435

[54] Huang M, Yan H, Heinz T F and Hone J 2010 Nano Lett. $104074-4079$

[55] Marinopoulos A G, Reining L, Rubio A and Olevano V 2004 Phys. Rev. B 69245419

[56] Pierucci D, Henck H, Avila J, Balan A, Naylor C H, Patriarche G, Dappe Y J, Silly M G, Sirotti F, Johnson A C et al. 2016 Nano Lett. 16 4054-4061

[57] Coy Diaz H, Avila J, Chen C, Addou R, Asensio M C and Batzill M 2015 Nano Lett. 15 1135-1140

[58] DaSilva A M, Jung J, Adam S and MacDonald A H 2015 Phys. Rev. B 91245422 